\title{
Correlated Analyses of Corroded Aluminum Alloy with a New SPM and FIB-SEM Linkage System
}

A. Morikawa ${ }^{1 *}$, T. Aiso ${ }^{1}$, M. Iyoki $^{1}$, T. Yamaoka ${ }^{1}$, T. Sato ${ }^{1}$, H. Suzuki ${ }^{2}$ and C. Kamiya ${ }^{1}$

1. Science Systems Product Division, Hitachi High-Technologies Corporation, Ibaraki, Japan.

2. Hitachi High-Tech Science Corporation, Shizuoka, Japan.

* Corresponding author: akinari.morikawa.jy@hitachi-hightech.com

Electric, magnetic or mechanical properties of materials have tight relation with surface and interior three dimensional (3D) structures of them. Thus, understanding the correlation of properties and material structure is crucial to develop and to improve high-performance materials. The SPM (Scanning Probe Microscopy) is technique for quantitative atomic-level measurement of specimen surface feature with electrical or mechanical properties simultaneously. While the SEM (Scanning Electron Microscopy) is technique for observation of surface structure, composition, crystalline and element distributions. Recently, 3D structural analysis method (reconstructed from serial section images can be collected by repetition of FIB slice and SEM observation/analysis) [1] is often used especially with FIB (Focused Ion Beam) and SEM composite instrument.

Although importance of interpreting correlation between the results of both microscopies was emphasized, practicing correlative measurements for randomly located micro-sized structure using plural instruments have been difficult. An SEM-SPM correlative microscopy system: SÆMic [2] for investigating identical position was developed and correlative measurements of surface features and surface electric properties became easier and practical. However, the SEM-SPM correlative analysis could not provide relation with specimen internal structure. In this study, newly developed linkage system for SPM and FIB-SEM specimen coordinates was evaluated by correlating surface electrical property by SPM and 3D reconstructed structure with an FIB-SEM instrument, ETHOS NX5000 [3].

The specimen corroded aluminum alloy (A6063) plate was prepared as follows; resin embedding a trimmed $\mathrm{Al}$ alloy plate, polishing the embedded plate, ion-milling the polished surface with a Hitachi ArBlade5000, finally the flattened surface was corroded by letting drops of $1 \% \mathrm{NaCl}$ solution on it for 30 minutes. Topographic images and surface potential images before and after the corrosion process were acquired with a Hitachi AFM5500M in KPFM (Kelvin Probe Force Microscopy) mode. Then, the specimen was loaded in the ETHOS NX5000 to acquire serial cross-sectional SEM images and EDX maps. To find the same field of view with the SPM, a compatible sample holder with alignment marks was used. Size of image acquisition area was $20 \mu \mathrm{m}$ (width) x $20 \mu \mathrm{m}$ (height), $19 \mu \mathrm{m}$ (depth) and $50 \mathrm{~nm}$ (slice pitch) and 380 frames were acquired in total. Acquired serial cross section SEM/EDX images were 3D reconstructed with Media Cybernetics, Image-Pro 3D Module software. The reconstructed result from EDX maps were overlaid on the surface potential images to compare correlation.

Figure 1 shows correlated KPFM images of the aluminum alloy surface before and after the corrosion. Regions of which potential is lower than the matrix are recognized to be on and diffused annularly around Fe inclusions due to the corrosion. Figure 2 shows a set of sectional SEM image (a), O-K (b), Al$\mathrm{K}$ (c), and Fe-L (d) EDX maps in sequentially acquired data with the FIB-SEM. Oxygen is detected at region of the surface and around the inclusion. Figure 3 shows $3 \mathrm{D}$ reconstructed images from O-K and Fe-L maps. The bird's view and top view reveal that oxygen is concentrated around inclusions, and 
lower potential regions measured with KPFM are coincided with oxygen detected regions. Also oxygen distribution is confined at the surface of specimen.

From these results, the SPM and FIB-SEM linkage system enables to measure physical properties and 3D structural analysis at the same field of view, and it was confirmed that analytical method with it is effective to understand correlation with the potential, composition and structure in analyses of metallic corrosion.

\section{References:}

[1] K Ohta et al., Micron 43 (2012), p. 612.

[2] A Miyaki et al., IMC2018, Boston, Oct31-Nov2, 2018

[3] S Torikawa et al., Proceedings of the 74th Annual meeting of The Japanese Society of Microscopy (2018), p.48.

\section{Before Corrosion}

(a)

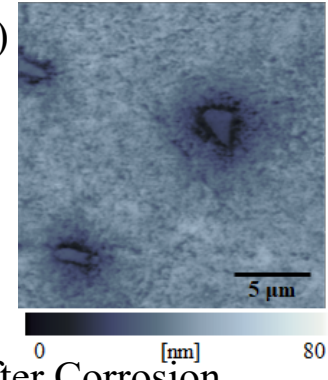

After Corrosion

(b)

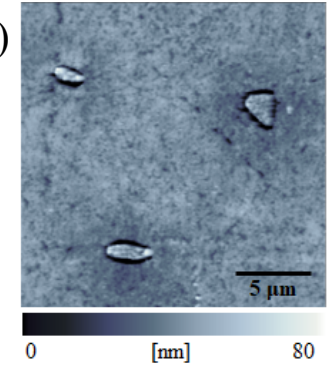

(c)

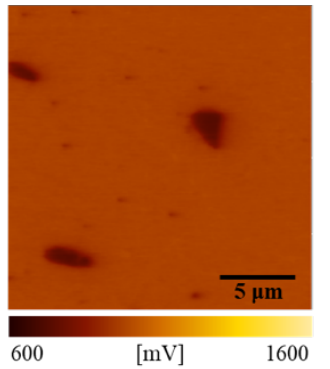

(d)

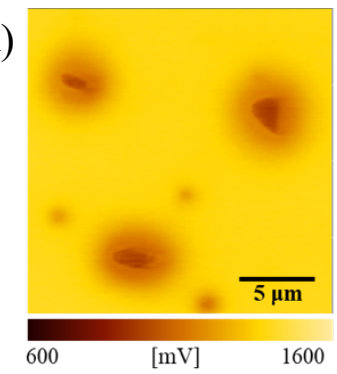

Figure 1. KPFM results before and after the corrosion: Topography (a), (b) and Surface potential (c), (d) images
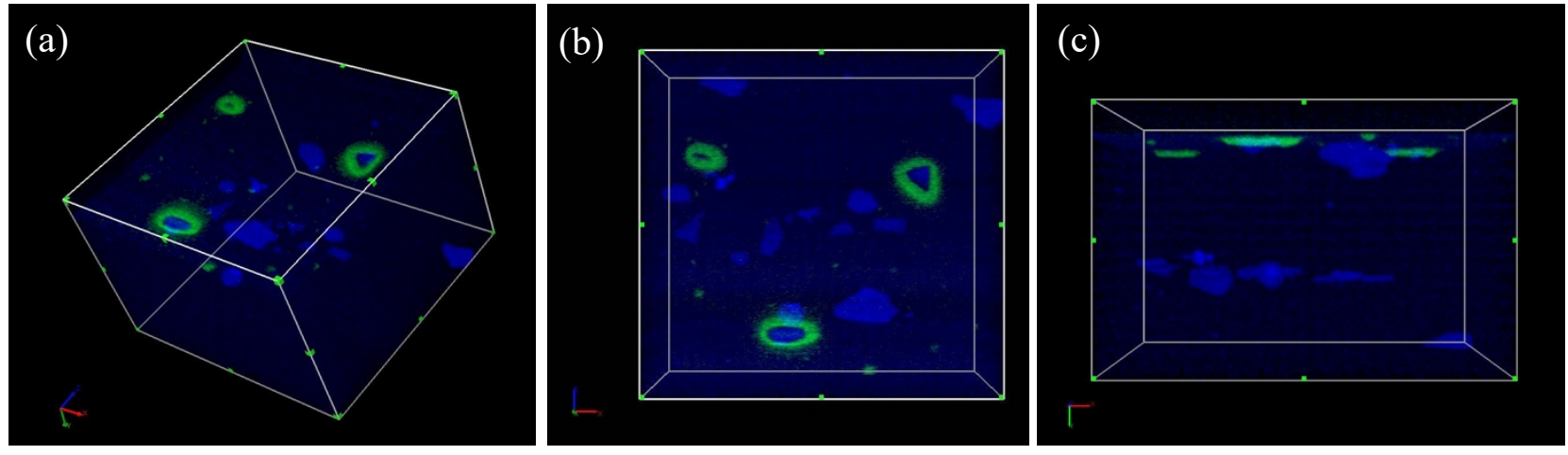

Figure 3. 3D reconstruction images of O-K (green) and Fe-L (blue) EDX maps

(a) Bird's view, (b) Top view, (c) Side view. 